\title{
"I'll Have One of Each": How Separating Rewards into (Meaningless) Categories Increases Motivation
}

\section{Citation}

Wiltermuth, S., and F. Gino. "'I'll Have One of Each': How Separating Rewards into (Meaningless) Categories Increases Motivation." Journal of Personality and Social Psychology (forthcoming).

\section{Permanent link}

http://nrs.harvard.edu/urn-3:HUL.InstRepos:10018933

\section{Terms of Use}

This article was downloaded from Harvard University's DASH repository, and is made available under the terms and conditions applicable to Open Access Policy Articles, as set forth at http:// nrs.harvard.edu/urn-3:HUL.InstRepos:dash.current.terms-of-use\#OAP

\section{Share Your Story}

The Harvard community has made this article openly available.

Please share how this access benefits you. Submit a story.

\section{Accessibility}




\title{
Running Head: CATEGORIES AND MOTIVATION
}

\author{
"I'll Have One of Each": \\ How Separating Rewards into (Meaningless) Categories Increases Motivation
}

Scott S. Wiltermuth

University of Southern California

Francesca Gino

Harvard University

Forthcoming, Journal of Personality and Social Psychology

The authors gratefully acknowledge comments on earlier drafts and helpful suggestions from

Drew Carton, Nate Fast, Cassie Mogilner, and Don Moore. Please address correspondence by email towiltermu@usc.edu. 


\begin{abstract}
We propose that separating rewards into categories can increase motivation, even when those categories are meaningless. Across six experiments, people were more motivated to obtain one reward from one category and another reward from another category than they were to obtain two rewards from a pool that included all items from either reward category. As a result, they worked longer when potential rewards for their work were separated into meaningless categories. This categorization effect persisted regardless of whether the rewards were presented using a gain or loss frame. Using both moderation and mediation analyses, we found that categorizing rewards had these positive effects on motivation by increasing the degree to which people felt they would "miss out" if they did not obtain the second reward. We discuss implications for research on motivation and incentives.
\end{abstract}

Keywords: Categories; Incentives; Motivation; Regret; Fear of Missing Out; Decision Making 


\section{"I'll Have One of Each":}

\section{How Separating Rewards into (Meaningless) Categories Increases Motivation}

Researchers across the social sciences have long sought to understand how to foster individual motivation. Much of this research has highlighted mechanisms that either increase or make salient the monetary or non-monetary benefits that people can obtain by applying effort. For instance, prior work has found that people become more motivated when the prosocial impact of their job is highlighted (Grant, 2007; 2008), when their work is imbued with task identity or task significance (Hackman \& Oldham, 1976), or when they come to see the performance of a task as central to their own identity (Koestner \& Losier, 2002). Setting concrete goals and providing meaningful rewards contingent upon the achievement of those goals can also increase individual motivation (Locke, 1968; Locke \& Latham, 2002; see Sansone \& Harackiewicz, 2000, for a review).

Drawing on research on the psychological principles of categorization (Rosch, 1973; Rosch \& Lloyd, 1978; Rosch, Mervis, Gray, Johnson, \& Boyes-Braem, 1976) and regret theory (Bell, 1982; 1983; Loomes \& Sudgen, 1982), we examine how categories of rewards influence individuals' motivation to exert task effort. We propose that grouping rewards into categories can increase the effort people apply toward goals, even when those categories are constructed arbitrarily, because categorizing incentives can increase the extent to which participants feel that they would be "missing out" on something if they failed to obtain a second reward. In illustrating this, our research goes beyond showing that people exhibit diminishing sensitivity to rewards in a single category and establishes a novel mechanism by which categorizing rewards enhances motivation.

Our investigation aims to establish that even factors that should not rationally affect the amount of effort people apply toward attaining incentives may in fact do so. By 
examining the effects of dividing rewards into meaningless categories on individual motivation, we contribute to research on the role psychological tendencies play in explaining the relationship between incentives and motivation. A more nuanced understanding of the impact of these psychological tendencies could benefit not only scholars interested in motivation but also parents, educators, managers, and anyone else who seeks to motivate others through rewards. Of course, such an understanding might also allow people to manipulate others into working more than they would otherwise deem optimal.

\section{Incentives and Motivation}

Motivation is defined as the driving force of directed activity that causes a person to act (Deci \& Ryan, 1985; Lewin, 1935; Ryan \& Deci, 2000). It is "the contemporary (immediate) influence on direction, vigor, and persistence of action” (Atkinson, 1964: 2). Several theories have been proposed to explain the process of motivation. Many of these theories assume that an individual's level of motivation depends directly on the expected consequences attributable to applying effort. Vroom's (1964) expectancy theory of motivation, for example, posits that people decide to behave in a particular manner because they expect the consequences of that behavior to be more desirable than the consequences of alternative behaviors. The theory holds that people consider both the likelihood of attaining the desired consequence and the expected valence of the consequence when deciding to apply effort. Incentive theory (Killeen, 1979; 1982; McDowell \& Kessell, 1979) also holds that the expected consequences of effort shape how motivated people are to work toward a goal. Indeed, most economic reasoning assumes that rewards can effectively motivate behavior (Laffont \& Martimort, 2002).

While such theories explain much about how incentives affect behavior, their ability to predict how incentives affect behavior is far from complete. Indeed, numerous scholars have asserted that expected utility theory would more accurately reflect behavior if it 
accounted for regret (e.g., Bell, 1982; Loomes, 1988; Loomes \& Sugden, 1982), which is a "negative, cognitively based emotion that we experience when realizing or imagining that our present situation would have been better, had we decided differently" (Zeelenberg, 1999, p.94). Bell (1982) and Loomes and Sugden (1982) each put forth regret theory, which assumes that the expected utility depends not only on the pain and pleasure associated with the outcomes of a decision, but also on the regret experienced (or anticipated) by comparing that outcome to those that would have resulted from different decisions. Providing support for this enhancement of utility theory, scholars have repeatedly found that the anticipation of regret can lead people to behave in ways designed to minimize the potential for subsequent regret (Loomes, Starmer, \& Sugden, 1992; Ritov, 1996; Zeelenberg, Beattie, van der Pligt, \& de Vries, 1996; Zeelenberg \& Beattie 1997; Zeelenberg, van Dijk, \& Manstead, 1998; but see Battalio, Kagel, \& Jiranyakul, 1991, and Harless, 1992).

Although scholars often draw upon regret theory to examine decision-making under uncertainty (e.g., Larrick \& Boles, 1995) and decision-making in consumer contexts (e.g., Simonson, 1992), little research has drawn upon regret theory to explain how much effort people will apply in pursuit of incentives. Yet, the degree to which people anticipate feeling regret for foregone incentives may well predict motivation in ways not predicted by current theories of motivation - particularly when multiple incentives are available and people may construe these incentives as falling into different categories. The present research explores this possibility, examining specifically how the categorization of non-monetary incentives may affect people's motivation by affecting their level of anticipated regret. We call this anticipated regret "fear of missing out."

\section{Categories and Anticipated Regret}

People cognitively construct categories using a basic level of processing (Rosch, 1975). This occurs because categories provide a great deal of information while allowing 
people to preserve their finite cognitive capacities (Rosch, 1978). In particular, categories allow people to identify differences between items very quickly (Heit \& Rubinstein, 1994; Lassaline, 1996; Rosch, 2002; Sloutsky, 2003). Because categories allow people to quickly sort items into similar and dissimilar categories, and because people believe that every act of communication conveys information (Clark, 1985; Grice, 1975), people tend to assume that items labelled as belonging to the same category are more similar than are items labelled as belonging to different categories (Mogilner, Rudnick, \& Iyengar, 2008).

We propose that the categorization of incentives has important implications for decision-making and motivation. Mogilner et al. (2008) demonstrated that this phenomenon has implications for consumers' perceptions and behavior. Using both field and laboratory experiments, the researchers found that increasing the number of categories available at the moment of choice (e.g., categories that partitioned an assortment of magazines) led to greater feelings of self-determination, which is the degree to which people experienced a sense of choice when making their purchasing decision. In turn, these greater feelings of selfdetermination increased satisfaction with one's purchase. The results were obtained even when the categories were completely arbitrary. While Mogilner et al. (2008) focused on how the presence of categories of items influence the choice people make when choosing one of the available items, here we examine how dividing potential rewards for performance into categories affect people's motivation to exert effort on the tasks that they are facing.

In this context, we argue, separating rewards into arbitrary categories is likely to increase the extent to which people fear they would be missing out by not attaining every category of reward. When rewards are uncategorized, people are unlikely to feel that they would miss out on the best reward available if they earned only one reward. Because people would not consider themselves to be missing out on obtaining the best reward available if they obtained only one reward, they would not likely anticipate that they would experience 
much regret from failing to obtain a second reward. However, when rewards are categorized into multiple categories, people may want to obtain a reward from each of the available categories in order to keep from feeling as if they are missing out on something. In short, they would anticipate greater regret. To avoid the negative emotional impact of failing to obtain a reward, people are likely to exert more effort when rewards are divided into multiple categories than when they are not. Fear of missing out can be a powerful motivating force. To wit, fear of missing out can motivate managers to work more hours than they otherwise would (Rutherford, 2001), college students to spend seemingly endless hours on the Internet (Kandell, 1998), athletes to return to competition prematurely after injury (Tracey, 2003), and investors to create speculative bubbles (Kindelberger, 1978/1989). We therefore propose that categorizing incentives increases people's motivation to obtain a second reward.

\section{Distinct Reference Points as an Alternative Mechanism}

Categorizing incentives may increase people's motivation to obtain multiple rewards because of an alternative mechanism that we account for in our research. It may lead people to consider multiple rewards as belonging to separate mental accounts (Thaler, 1985, 1990, 1999), which are defined as frames that specify “(i) the set of elementary outcomes that are evaluated jointly and the manner in which they are combined, and (ii) a reference outcome that is considered neutral or normal" (Kahneman \& Tversky, 1981: 456). Considerable research (e.g., Thaler \& Johnson, 1990) supporting Kahneman \& Tversky's (1979) prospect theory has demonstrated that people's sensitivity to increases in gains or losses diminishes with the magnitudes of those gains and losses, such that the gain function is concave and the loss function is convex (see Figure 1). ${ }^{1}$ If categorizing rewards leads people to view those rewards as belonging to separate mental accounts, it should also lead them to use separate

\footnotetext{
${ }^{1}$ Prospect theory also holds that people are loss-avoidant, such that the loss function is steeper than the gain function. This aspect of the theory is not particularly relevant for our predictions.
} 
reference points when evaluating those rewards. Given the concavity of the gain function, people should therefore be more sensitive to increases in gains that involve distinct mental accounts than they should be to increases in gains within a single mental account. As such, categorizing rewards should lead people to be more motivated to pursue a second reward. In our experiments, we test whether this alternative mechanism may explain the link between categorization and motivation.

In short, we propose that separating gains into categories increases the degree to which people feel as though they would be missing out if they failed to obtain rewards from each category. Specifically, we propose that simply labelling potential rewards as belonging to different categories can increase motivation, even when those categories do not objectively differ. For example, people might be more motivated to obtain both a box of candy and a box of popcorn if these items were said to be from different categories of goods than if they were said to be from a single category. Thus, we seek primarily to demonstrate how the simple act of categorizing incentives increases their impact on individual motivation, even when categorization does not increase the objective value of the incentives.

\section{Overview of the Present Research}

In six experiments, we test the hypothesis that separating incentives into categories can increase the effort people exert to attain those incentives, even when those categories are arbitrarily constructed. Furthermore, we examine how fear of missing out explains the relationship between categorizing incentives and increased motivation. Finally, we rule out alternative mechanisms that could potentially explain our effects. In Experiment 1, we examine whether splitting incentives into arbitrary categories leads people to apply more effort toward a goal. In the next two experiments, we replicate the results of Experiment 1 using a loss rather than a gain frame (Experiment 2) and a different measure of motivation, namely self-reported task performance (Experiment 3). In Experiments 4, 5 and 6, we test 
whether categorizing rewards increases motivation by creating a fear of missing out and rule out the alternative mechanism of establishing distinct reference points. In Experiment 4, we examine whether the categorization-of-rewards effect is strongest when increased effort can largely eliminate the fear of "missing out" associated with failing to obtain a second reward. Experiment 5 demonstrates that fear of missing out mediates the link between categorization and increased motivation. Finally, Experiment 6 employs both mediation and moderation analyses to provide further evidence for the process by which categorization increases effort.

\section{Experiment 1: Two Categories or a Single Category}

In Experiment 1, we tested whether splitting incentives into arbitrary categories lead people to apply more effort toward a reward. We did so by manipulating whether items purchased from a local dollar store were portrayed as belonging to a single category or to two categories. The items available in the two conditions were the same. In testing our main hypothesis, we attempted to move beyond current research showing that people respond differently to financial and non-financial incentives (e.g., Heyman \& Ariely, 2004) to show that the framing of non-financial incentives can and does affect individual motivation.

\section{Method}

Participants and design. Sixty-three undergraduate business students (56\% female; $\left.M_{\text {age }}=21.0\right)$ at a large, private university on the West Coast of the United States participated in the study in exchange for course credit. Participants were randomly assigned to one of two experimental conditions: categorization and no-categorization.

Procedure. We instructed participants that they would be transcribing a number of sections of typewritten text to help us prepare for a future study in which we would examine how handwriting can affect the perceptions people form of others. Participants were told they could spend as much time or as little time transcribing the sections of text as they liked. They then examined their potential rewards, which were placed in two 55-quart storage containers. 
The rewards were not sorted into specific categories; rather, there was a mix of stationery and food items in each container. Thus, while in this study and most of the studies that follow the containers contained slightly different mixes of products, the differences in product mixes were not meaningful. Examples of rewards were: boxes of hot cocoa, packages of pens, calculators, notebooks, and animal crackers. Participants then read about the rewards they would accumulate for spending at least ten minutes and at least twenty minutes transcribing the sections of text. Participants then began transcribing the sections of text. After they decided to stop transcribing, participants selected their reward(s). As their last task, participants completed a short questionnaire.

In this and all following experiments except Experiment 5, the experimenter was blind to participant condition. In Experiment 5, it was not possible to keep the experimenter blind to participant condition; however, the experimenter in Experiment 5 was unaware of the study hypotheses.

Categorization manipulation. We manipulated whether participants perceived the rewards as belonging to two distinct categories or only one. In the categorization condition, participants read:

There are two categories of rewards you can earn by spending time transcribing these sections of text. Category 1 is in the Purple Storage Container and Category 2 is in the Clear Storage Container. If you spend ten minutes transcribing these sections of text, you will be allowed to take home one of the items from either the Purple Storage Container or the Clear Storage Container. It will be your choice. If you spend twenty minutes transcribing these sections of text, you will also be allowed to take one of the items home from the other storage container. So, you will be able to take home an item from each of the two categories if you spend twenty minutes transcribing the sections of text.

In the no-categorization condition, participants read:

There are rewards you can earn by spending time transcribing these sections of text. If you spend ten minutes transcribing the sections of text, you will be allowed to take home one item. If you spend twenty minutes transcribing the sections of text you will be allowed to take home a second item. So, you will be able to take home two items if you spend twenty minutes transcribing these sections of text. 
Thus, in the no-categorization condition, participants actually had more choice regarding the rewards they could take, as they could have chosen two rewards from the same container. Participants in both conditions visually inspected the rewards before starting the transcription task. The items in each storage container were of equal monetary value.

Measures. The likelihood of participants transcribing sections of text for a full twenty minutes served as the primary dependent variable. After the transcription task, participants indicated on a seven-point Likert-type scale $(1=$ Not at all, to $7=$ Very much $)$ how motivated they were to earn the first reward, how motivated they were to earn the second reward, and how much they enjoyed the task.

\section{Results}

We first used a logistic regression to examine the effect of categorization condition on participants' likelihood of working for the full twenty minutes required to claim two rewards. Participants in the categorization condition were more likely to transcribe for the full twenty minutes $(34.4 \%)$ than were participants in the no-categorization condition $(9.7 \%), \mathrm{B}=0.79$, $\mathrm{SD}=.36, \mathrm{Wald}=4.96, \operatorname{Exp}(\mathrm{B})=2.21, p=.03$. They also reported that they were more motivated to obtain the second reward $(M=4.22, S D=2.21)$ than did participants in the nocategorization condition $(M=3.07, S D=1.95), t(60)=2.17, p=.03, d=.55$. We note that participants' self-reported motivation to obtain the second reward correlated significantly with their likelihood to transcribe for the full twenty minutes, $r(62)=.36, p<.01$.

Participants in the categorization condition also reported enjoying the task more $(M=$ $2.97, S D=1.49)$ than did those in the no-categorization condition, $(M=2.20, S D=1.27)$, $t(59)=2.16, p=.04, d=.52$. However, task enjoyment did not mediate the link between categorization condition and likelihood of working for the full twenty minutes, nor did it mediate the link between categorization condition and self-reported motivation to obtain the second reward. 
Importantly, we found no differences across conditions in likelihood to transcribe for the ten minutes required to obtain the first reward $(p>.40)$, nor in motivation to obtain the first reward $(p>.35)$.

\section{Discussion}

The results of Experiment 1 provide support for the categorization-of-rewards effect we hypothesized: participants spent more time working on the transcribing task and reported feeling more motivated to obtain a second reward in the categorization condition than in the no-categorization condition, even though the categories of rewards created in this study were completely arbitrary. Interestingly, participants also reported enjoying the task more in the categorization of rewards condition.

\section{Experiment 2: Loss Frame and Valuation of Rewards}

Experiment 1 participants were more motivated and worked longer when their rewards ostensibly came from two categories rather than from a single category. As such, participants derived more subjective value from categorized rewards than they did from noncategorized rewards even though the objective value of the rewards was equivalent. To test the robustness of these findings, Experiment 2 employs a loss frame. Specifically, it examines whether participants would be willing to work longer to avoid losing rewards from two distinct categories than they would to avoid losing multiple rewards from a single category. Experiment 2 also examines if the categorization effect persists when people actively calculate the value of the incentives before they engage in the given task. The valuation of the incentives may attenuate or eliminate the effect of categorization if it leads people to focus on the financial value of the incentive. We expected that it would not.

\section{Method}

Participants and design. One hundred thirty-one business students (56\% female; $\left.M_{\text {age }}=20.6\right)$ at a large, private university on the West Coast of the United States participated 
in the study in exchange for course credit. Participants were randomly assigned to one of four experimental conditions from a 2 (categorization of rewards) X 2 (valuation of rewards) between-subjects design.

Procedure. Participants completed the same task used in Experiment 1. However, in this study, participants selected their potential rewards before embarking on the transcription task. Additionally, we introduced a second manipulation to examine whether categorization increases effort even when participants are first asked to estimate the monetary value of potential rewards. We varied whether participants calculated the monetary value of their rewards before they engaged in the transcription task. After the transcription task, participants rated how motivated they were to earn the second reward (on a 7-point scale, ranging from 1 $=$ Not at all, to $7=$ Very much).

Categorization manipulation. We manipulated whether participants perceived the rewards as belonging to two distinct categories or only one. In the categorization condition, participants first read:

There are two categories of rewards that you earn by doing this experiment. Category 1 is in the Purple Storage Container and Category 2 is in the Clear Storage Container. Please go to the front of the room and select an item that you would like to take home with you from each of these two categories.

They were then told:

If you work for at least twenty minutes transcribing the sections of text, you will be able to keep an item from each of the two categories. If you spend between ten and twenty minutes transcribing the sections of text, you will have to return an item from one of the two categories but will be allowed to keep the item from the other category. If you spend less than ten minutes transcribing the sections of text, you will have to return the items from both categories. So, spending twenty minutes transcribing the sections of text would enable you to take home an item from each of the two categories.

In the no-categorization condition, participants read:

There are rewards that you earn by doing this experiment. If you work for at least twenty minutes transcribing the sections of text, you will be able to keep both of the items you have selected. If you spend between ten and twenty minutes transcribing the sections of text, you will have to return one of the items but will be allowed to keep one of the items. If you spend less than ten minutes transcribing the sections of text, you 
will have to return both of the items you selected. So, spending twenty minutes transcribing the sections of text would enable you to keep both items.

We reminded participants in both conditions that they could spend as much or as little time as they liked on the transcription task. As in Experiment 1, the items in each storage container were of equal monetary value.

Valuation manipulation. Participants in the valuation condition estimated the monetary value of each of the items that they selected. Participants in the no-valuation condition did not.

Dependent variables. The primary dependent variable was participants' likelihood of transcribing sections of text for a full twenty minutes. We also examined participants' selfreported motivation to earn the second reward.

\section{Results}

We first conducted a logistic regression to examine the effect of categorization condition and valuation condition on participants' likelihood of working for the full twenty minutes required to retain two rewards. We found a significant main effect for categorization condition $(\mathrm{B}=0.70, \mathrm{SD}=.36, \mathrm{Wald}=3.70, \operatorname{Exp}(\mathrm{B})=2.01, p=.05)$. As Table 1 illustrates, participants in the categorization condition were more likely to transcribe for the full twenty minutes $(49.3 \%)$ than were participants in the no-categorization condition $(32.8 \%)$. The valuation condition did not significantly affect participants' likelihood of working the entire twenty minutes $(p>.70)$.

Consistent with this finding, participants in the categorization condition reported being more motivated to obtain the second reward $(M=3.76, S D=2.27)$ compared to participants in the no-categorization condition $(M=3.16, S D=1.78), \mathrm{t}(127)=2.02, p=.05, d$ $=.29$. Participants' self-reported motivation to obtain the second reward correlated significantly with their likelihood to transcribe for the full twenty minutes, $r(130)=.36, p<$ 
.01 . Together, these results provide further support for our hypothesis regarding the effects of categorizing rewards on individual motivation.

We did not find significant differences across condition in participants' likelihood to transcribe for the ten minutes required to obtain the first reward, $p>.90$.

\section{Discussion}

Experiment 2 tested the robustness of the categorization effect by using a loss frame. Providing further support for our main hypothesis, participants exerted more effort when their rewards were split into meaningless categories than when they were not, indicating that their motivation was influenced by the categories of rewards. Importantly, this categorization effect persisted when participants estimated the monetary value of the incentives before engaging in the task.

\section{Experiment 3: Getting One of Each}

We suggested that categorizing rewards increases the extent to which people feel that they would miss out on something if they failed to obtain a second reward. To provide support for this mechanism, Experiment 3 tests whether the strength of the categorization effect differs depending upon whether increased effort could eliminate people's fear of missing out on something. We predicted that people who could expend effort to eliminate the fear of missing out on something would be more motivated than would participants whose efforts could not eliminate such fear. In essence, we propose that the opportunity to eliminate the fear of missing out on something provides motivation not present in conditions in which participants have no opportunity to eliminate the fear of missing out on something. Thus, if people could only choose two rewards out of more than two categories as compensation for their performance, we would expect them to be less motivated to exert effort in the given task than when they could choose a reward from each of two categories. In the latter case, we would expect the chance to eliminate the fear of missing out on something to result in higher 
motivation than would be evident in the former case. In Experiment 3, we consequently employ conditions in which people's increased motivation and effort can enable them to earn rewards from each possible category, and, in doing so, greatly attenuate the risk of regret caused by missing out on something.

In particular, we test whether categorizing rewards increases performance when that increased effort can allow people to achieve all categories of rewards rather than only some of the categories. We reason that the relationship between performance and the attenuation of anticipated regret is stronger when the effort enables the person to obtain a reward from all available categories.

Experiment 3 also allows us to test for the role of two alternative mechanisms that can explain the effects demonstrated in Experiments 1 and 2. First, this third experiment includes a measure of perceived variety to examine whether it influences motivation. Previous work has demonstrated that categorization can lead to increased satisfaction in consumer choices by increasing perceived variety (Mogilner et al., 2008). In a similar manner, categorization may lead to increased motivation through the same mechanism when the object of categorization is potential rewards for performance. That is, people may be more motivated when people perceive that there are a variety of rewards available to them than they would be when they perceive that the rewards available are all very similar. We test this possibility directly in Experiment 3 by including a measure of perceived variety.

Second, this experiment allows us to test for the role of establishing separate reference points, the alternative mechanism discussed in the introduction. According to this account, people treat rewards from different categories as belonging to different accounts and so the rewards are separately motivating. People treat rewards from the same category as belonging to the same account, causing them to devalue the second of the two potential rewards due to diminishing sensitivity. To rule out this alternative account, Experiment 3 includes three 
conditions (rather than just two as in our previous studies). Across conditions, we varied the number of rewards and categories of rewards available to participants. Specifically, in addition to a no-categorization condition, we included a two-of-two categories condition (where the second reward is from a second category out of two available), and a two-of-four categories condition (where the second reward is from a second category out of four available). We expected participants' effort to be higher in the two-of-two categories condition than in both the no-categorization condition and the two-of-four categories condition. Furthermore, we did not expect differences in participants' effort between the twoof-four categories condition and the no-categorization condition. Such a pattern of results would be consistent with our main hypothesis by showing that categorizing rewards improves motivation most dramatically when participants' performance allows them to attain rewards from each available category. Instead, if diminishing sensitivity is the correct explanation for the categorization-of-rewards effect, we would observe greater effort in the two-of-four categories condition relative to the no-categorization condition.

\section{Method}

Participants. One hundred seventy-two online participants $\left(48.9 \%\right.$ female; $M_{\text {age }}=$ 33.3, $S D=11.2$ ) recruited from Amazon.com's MTurk website participated in the experiment in exchange for $\$ 1$ plus the chance to earn additional prizes.

Procedure. We instructed participants that they would be alphabetizing groups of three fruit (e.g., pomegranate, raspberry, and mango). Before beginning the alphabetizing task, participants were presented with the lists of rewards from which they could choose if they alphabetized more groupings of fruit than $70 \%$ of participants. We instructed participants that they would be able to earn a second reward if they alphabetized more groupings of fruit than $90 \%$ of participants. 
Categorization manipulation. We manipulated how the rewards were categorized across three experimental conditions: no-categorization, two-of-two categories, and two-offour categories. Participants in the no-categorization condition read that they could choose one reward if they alphabetized more groupings of fruit than $70 \%$ of participants, and two rewards if they alphabetized more groupings of fruit than $90 \%$ of participants. Participants in the two-of-two categories condition read that they could choose a reward from either of the two groups of rewards if they alphabetized more groupings of fruit than $70 \%$ of participants, and that they could choose a reward from the second group if they alphabetized more groupings of fruit than $90 \%$ of participants. Those in the two-of-four categories condition read that they could choose a reward from one of the four groups of rewards if they alphabetized more groupings of fruit than $70 \%$ of participants and that they could choose a reward from a second group if they alphabetized more groupings of fruit than $90 \%$ of participants. Each group of rewards contained a mix of items similar to those used in our first two experiments, and participants were provided with a full list of the items in each group. We instructed participants in both the two-of-two categories condition and the two-of-four categories condition that we arbitrarily placed the items in each group, and told them that, as a result, each group of possible rewards contained similar items.

The alphabetizing task. Participants were asked to estimate how many groupings of three fruit they would alphabetize. They were free to use a value between zero and fifty as their estimate. Participants then alphabetized groupings of fruit, our measure of effort.

\section{Measures}

Dependent variable. The primary dependent variable of interest was the number of fruit groupings participants alphabetized.

Perceived variety. We asked participants about variety because we wanted to ensure that categorizing items did not increase their motivation by increasing the amount of variety 
participants perceived in the available rewards. Participants used a seven-point Likert-type scale (ranging from $1=$ not at all, to $7=$ very much) to respond to three items reflecting how much variety they perceived in the rewards $(\alpha=.71)$. The three items were: "How much variety was there in the rewards you could earn?", "How different would the second reward you choose be from the first reward you choose?" and "How similar were the rewards available to you?" (reverse coded).

\section{Results}

Reported performance. A one-way ANOVA revealed that the effect of categorization condition (no categorization vs. two-of-two categories vs. two-of-four categories) on participants' reported performance was marginally significant, $F(2,169)=$ $2.34, p=.10$.

Planned contrasts showed that participants in the two-of-two categories condition alphabetized more groupings $(\mathrm{M}=31.7, \mathrm{SD}=17.1)$ than did participants in the other two conditions combined $(\mathrm{M}=26.3, \mathrm{SD}=18.0), t(169)=1.96, p=.05, d=.31$ and participants in the no categorization condition alone $(\mathrm{M}=24.7, \mathrm{SD}=17.6), t(169)=2.15, p=.03, d=.41$. The number of groupings alphabetized by those in the two-of-four categories condition $(\mathrm{M}=$ $27.8, \mathrm{SD}=18.4)$ did not significantly differ from that of participants in the no-categorization condition $(t(169)=1.24, p=.22, d=.17)$, or in the two-of-two categories condition, $t(169)=$ $0.91, p=.36, d=.22$.

Perceived variety. Categorization did not influence participants' perceived variety, $F(2,169)=1.83, p=.16$. Importantly, participants did not perceive greater variety in the twoof-two categories condition $(M=4.10, S D=1.17)$ than they did in the no-categorization condition $(M=4.44, S D=1.31 ; t(169)=1.52, p=.13)$ or the two-of-four categories condition $(M=4.49, S D=1.10 ; t(169)=1.76, p=.08)$.

\section{Discussion}


As predicted, categorizing rewards increased the degree to which people anticipated they would miss out if they did not attain multiple rewards. When participants had the opportunity to obtain rewards from each of the available categories (i.e., in the two-of-two categories condition), their motivation increased as indicated by their higher performance. However, their motivation was not enhanced when the presence of multiple categories of rewards was not accompanied by the possibility of receiving rewards from each of the available categories (i.e., in the two-of-four categories condition). Thus, results were consistent with the idea that categorizing rewards increases effort by instilling anticipated regret about missing out on rewards. The results were neither consistent with the alternative account of increased variety, nor with the notion that categorizing rewards establishes separate reference points.

\section{Experiment 4: Mediation by Fear of Missing Out}

In Experiment 3, we used multiple conditions to test for the role of the anticipated regret associated with missing out in explaining the link between categorization of rewards and individual motivation. In Experiment 4, we provide further evidence for the posited psychological mechanism by measuring fear of missing out directly. Specifically, we ask participants about feelings of missing out before they perform an anagram task that we use to assess their performance. We do so because we believe it is the anticipation of missing out that drives motivation. We therefore seek to show that a heightened fear of missing out mediates the relationship between categorization of rewards and increased motivation.

\section{Method}

Participants and design. One hundred thirty-nine online participants (65\% female; $\left.M_{\text {age }}=30.7, S D=10.3\right)$ recruited from Amazon.com's MTurk website participated in the experiment in exchange for $\$ 2$ plus the opportunity to earn additional prizes. We randomly assigned participants to one of two conditions: no categorization and categorization. 
Procedure. We instructed participants that they would be forming words out of a series of scrambled letters, an anagram task we adapted from Schweitzer et al. (2004). Specifically, participants saw an example consisting of the letters ISTEBOM and were told to write down, on a separate sheet of paper, as many words as they could form out of those letters in one minute, and then report the number of words they formed. After the example, participants completed six experimental rounds. In each round, they were given a different set of seven letters, and were asked to generate as many words as they could in the allotted one minute. In both conditions, participants simply reported the number of words they formed. Because they were not required to list those words, they had an opportunity to over-report their performance.

Categorization manipulation. Before beginning the anagram task, participants viewed the lists of potential rewards from which they could choose depending on their performance, and received instructions on how they could receive the rewards. Participants in the no-categorization condition read that they could choose one reward if their performance placed them in the top $30 \%$ of test takers, and two rewards if their performance placed them in the top $10 \%$ of test takers. Participants in the categorization condition read that they could choose a reward from either of the two categories of rewards available if their performance placed them in the top $30 \%$ of test takers, and that they could choose a reward from the second category if their performance placed them in the top $10 \%$ of test takers. Each category contained a mix of items similar to those we used in Experiments 1-3. We provided participants with a full list of the items in each category, but, differently from Experiment 3, we did not tell them explicitly that we assigned the items to the groups arbitrarily.

After viewing the potential rewards and before completing the anagram task, participants responded to the question "How much would you be missing out on something if 
you did not earn both rewards?" using a seven-point Likert-type scale $(1=$ Not at all, to $7=$ Very Much). This question measured fear of missing out.

Measures. Participants' reporting of how many words they formed served as the primary dependent variable. We used participants' self-reported fear of missing out as the mediating variable.

\section{Results}

As we expected, participants in the categorization condition indicated that they were more concerned about missing out on the second reward $(M=3.42, S D=1.70)$ than were participants in the no-categorization condition, $(M=2.78, S D=1.66), t(137)=2.23, p=.03$, $d=.38$. They also reported creating more words in the anagram task $(M=29.56, S D=17.30)$ than did participants in the no-categorization condition $(M=24.68, S D=12.15), t(137)=$ $1.94, p=.05, d=.33$.

We conducted a mediation analysis to determine whether fear of missing out mediated the relationship between categorization and higher reported performance on the anagram task. Participants in the categorization condition reported feeling greater fear of missing out than did those in the no-categorization condition, $\mathrm{B}=0.63, \mathrm{SE}=0.28, t(136)=2.23, p=.03$. The fear of missing out correlated positively with reported performance, $\mathrm{B}=1.52, \mathrm{SE}=0.75$, $t(136)=2.04, p=.04$. Accounting for the fear of missing out reduced the significant relationship between condition and reported performance $(\mathrm{B}=4.88, \mathrm{SE}=2.51, t(136)=1.94$, $p=.05)$ to non-significance, $\mathrm{B}=0.63, \mathrm{SE}=0.28, t(136)=2.23, p=.12$. A bootstrap analysis revealed that the $95 \%$ bias-corrected confidence interval for the size of the indirect effect excluded zero $(0.07,4.13)$, suggesting a significant indirect effect (MacKinnon et al., 2007; Preacher \& Hayes, 2004). Thus, fear of missing out mediated the relationship between the presence of categories of incentives and increased reported performance.

\section{Discussion}


Experiment 4 provides further evidence for the psychological mechanism explaining the robust link between categorization of rewards and increased motivation. We found that when rewards were divided into arbitrary categories, participants reported higher levels of performance and that fear of missing out mediated this link.

\section{Experiment 5: Further Evidence for the Mediating Role of Fear of Missing Out}

In Experiment 4, we measured motivation through self-reported performance. It is possible that our categorization manipulation affected participants' motivation to inflate their performance on the anagram task (i.e., to cheat) rather than their motivation to exert greater effort on the task. Since we did not have data to compare actual to reported performance, we could not rule out this possibility in Experiment 4. To address this potential confound, we conducted another experiment in which we measured motivation to exert effort on a task. Furthermore, in Experiment 5, we used a different manipulation for categories of rewards to assure that participants perceived the categories of rewards as meaningless. Specifically, we presented the categories of rewards to participants without referring to these categories explicitly. Instead, we placed the potential prizes in front of participants within either two or three containers.

\section{Method}

Participants. One hundred one students attending local universities in a city in the South-eastern United States $\left(57 \%\right.$ male; $\left.M_{a g e}=21.72, S D=3.21\right)$ participated in the study for \$7. We recruited the participants through an advertisement on a university-wide website.

Procedure and design. The experiment used a job application cover-letter editing task developed by Grant et al. (2007). We informed participants that we were collaborating with a local career center to enrich our knowledge of how to improve students' effectiveness in their job searches. Participants were told that the career center had provided actual cover 
letters from students who were searching for jobs and that their task was to edit a student's cover letter and provide feedback on how to improve it.

The experiment employed one between-subjects manipulation with two experimental conditions: two containers of potential rewards and three containers. At the beginning of the study the experimenter placed either two or three containers on a table in the center of the room, such that the containers were visible to all participants. As potential rewards, we used the same types of items as in our other studies. In this experiment, however, all items were initially in the same large basket. In both conditions, the experimenter dumped the rewards in the plastic containers (either two or three depending on the experimental condition) while saying, "Here are the potential rewards for completing the editing task, in addition to the monetary payment you received for participating in today's study.” The experimenter then told participants that if they spent ten minutes editing the cover letter, they would be allowed to take home one of the items of their choosing. If, instead, they spent twenty minutes or more working on the editing task, they would be allowed to take home two of the items of their choosing. Finally, the experimenter noted that the containers contained similar items, and asked participants to walk close to the table so that they could view the items before they started working on the editing task.

Next, participants received a student's job application cover letter in Microsoft Word, and were asked to introduce edits and provide feedback using the Track Changes feature. The experimenter informed participants that they could stop working on the task whenever they wanted or felt they were finished. The experimenter recorded the amount of time in minutes that participants spent on the task and informed them that we would use such information to calibrate future studies. Participants had timers on their individual desk so that they could easily keep track of time. The amount of time participants spent editing the cover letter served as our measure for effort (our dependent measure). 
Before participants engaged in the editing task and after viewing the potential rewards, they completed a short questionnaire which included a two-item measure for fear of missing out ("How much would you be missing out on something if you did not earn both rewards?" and "How much would you fear missing out on something if you did not earn both rewards?," $\alpha=.91)$. Participants indicated their answers for each item using a 7-point scale (from $1=$ Not at all, to $7=$ Very much). We used this measure as our mediator in the analyses we present below.

\section{Results}

We first examined the effect of our manipulation (i.e., the presence of two vs. three containers containing potential rewards) on participants' effort. As predicted, and consistent with the results of the previous studies, participants in the two-containers condition spent more time working on the editing task $(M=18.20 \mathrm{~min}, S D=5.00)$ than did participants in the three-containers condition $(M=15.80, S D=4.96), t(99)=2.42, p=.018, d=.48$. Consistent with this result, a larger percentage of participants spent 20 minutes or more working on the editing task in the two-containers condition (62\%) than in the three-containers condition $(41.2 \%), \chi^{2}(1, N=101)=4.38, p=.036$. In addition, participants indicated that they were more concerned about missing out on something if they did not earn both rewards in the twocontainer than in the three-container condition $(M=4.28, S D=1.40$ vs. $M=3.59, S D=$ 1.28), $t(99)=2.59, p=.011, d=.51$.

Next, we examined whether participants' fear of missing out mediated the effect of our manipulation on the amount of time participants spent working on the editing task. The effect of two versus three containers of rewards was reduced to marginal significance (from $\beta$ $=.24, t=2.42, p=.018$ to $\beta=.17, t=1.77, p=.08$ ) when fear of missing out was included in equation, and fear of missing out was a significant predictor of participants' effort $(\beta=.25, t$ $=2.50, p=.014)$. A bootstrap analysis showed that the $95 \%$ bias-corrected confidence 
interval for the size of the indirect effect excluded zero $(.088,1.68)$, suggesting a significant indirect effect (MacKinnon et al., 2007; Preacher \& Hayes, 2004).

Results examining participants' likelihood to work 20 minutes or more on the editing task were similar. The effect of the categories manipulation on likelihood to work for at least 20 minutes was reduced to non-significance (from $\mathrm{B}=0.85, \mathrm{SD}=.41$, Wald $=4.32, \operatorname{Exp}(\mathrm{B})$ $=2.33, p=.038$ to $\mathrm{B}=0.64, \mathrm{SD}=.43$, Wald $=2.27, \operatorname{Exp}(\mathrm{B})=1.90, p=.13)$ when we included fear of missing out as a mediator. Moreover, fear of missing out was a significant predictor $(\mathrm{B}=0.35, \mathrm{SD}=.16, \mathrm{Wald}=4.67, \operatorname{Exp}(\mathrm{B})=1.42, p=.031)$. Also in this case, a bootstrap analysis revealed that the $95 \%$ bias-corrected confidence interval for the size of the indirect effect excluded zero $(0.02,0.76)$, suggesting a significant indirect effect (MacKinnon et al., 2007; Preacher \& Hayes, 2004). Together, these result show that fear of missing out mediated the relationship between categorizing rewards into meaningless categories and participants' enhanced motivation to exert effort on the editing task, even if there was no explicit mention of categories.

\section{Discussion}

The results of Experiment 5 further support our main hypotheses regarding the relationship between categorizing rewards and enhanced motivation, and the role of fear of missing out in explaining this link. Using a procedure that assured that participants perceived the categories of rewards as meaningless, we found that participants worked longer on an editing task and more strongly feared missing out on a second reward for their performance when they could attain two rewards of their choosing among rewards placed in two rather than three containers.

\section{Experiment 6: The Same Items in Each Container}

We conducted a final experiment to further corroborate our hypothesis that categorizing incentives can increase motivation by instilling feelings of anticipated regret 
about missing out on incentives. In this experiment, we placed exactly the same items within each of the containers. As in Experiment 5, we also omitted the word "category" from the instructions to see if segregating rewards into categories would produce differences in effort even if we did not explicitly activate the construct of categories. Moreover, we asked all participants to note that the experimenter did not care how much time they spent on the transcription task. Finally, we included a condition in which participants could earn a reward from each of two of three available containers.

\section{Method}

Participants and procedure. One hundred thirty-one undergraduate students (51\% female; $M_{\text {age }}=20.3, S D=2.5$ ) participated in the experiment in exchange for course credit plus the chance to earn additional prizes. Participants were randomly assigned to one of three experimental conditions: two-of-two containers of rewards, combined containers, and two-ofthree containers. They completed the same transcribing task we used in Experiments 1 and 2. This time, however, participants received a reward for transcribing for five minutes, and two if they transcribed for at least ten minutes.

Categorization manipulation. We manipulated the presentation of rewards across three conditions. In the two-of-two containers condition, participants first read:

If you spend five minutes transcribing these sections of text, you will be allowed to take home one of the items from either the Purple Storage Container or the Clear Storage Container. It will be your choice. They contain similar items and you are free to peruse the items before engaging in the task. If you spend ten minutes transcribing these sections of text, you will also be allowed to take home one of the items from the other storage container. So, you will be able to take home an item from each of the two storage containers if you spend ten minutes transcribing the sections of text.

In the combined containers condition, participants read:

If you spend five minutes transcribing these sections of text, you will be allowed to take home one of the items from the storage containers. It will be your choice. The storage containers contain similar items. You are free to peruse the items before engaging in the task. If you spend ten minutes transcribing these sections of text, you will also be allowed to take home a second item. So, you will be able to take home two items if 
you spend ten minutes transcribing the sections of text.

In the two-of-three containers condition, participants read:

If you spend five minutes transcribing these sections of text, you will be allowed to take home one of the items from either the Purple Storage Container, the Clear Storage Container, or the Gray Storage Container. It will be your choice. The storage containers contain similar items. You are free to peruse the items before engaging in the task. If you spend ten minutes transcribing these sections of text, you will also be allowed to take home one of the items from one of the other storage containers. So, you will be able to take home an item from two of the three storage containers if you spend ten minutes transcribing the sections of text.

The written instructions reminded participants in all conditions that they could spend as much or as little time as they liked on the task.

\section{Measures}

Effort. The primary dependent variable was participants' likelihood of transcribing sections of text for a full ten minutes.

Anticipated regret. Before participants began transcribing, we asked them to use a seven-point scale (ranging from $1=$ Strongly disagree, to $7=$ Strongly agree) to rate their agreement with the statement that they would regret their decision if they had decided to work for 5 minutes and therefore did not receive the second reward item.

Perceived experimenter concern. To ensure that participants' inferences about experimenter concerns did not drive our effects, we asked participants how much they thought the experimenter cared about how much time they spend on the transcription task.

\section{Results}

Effort. We conducted a logistic regression to examine the effect of condition on participants' likelihood of working for the full ten minutes required to retain two rewards. We used the two-of-two containers condition as the baseline condition and used dummy variables to represent the combined containers condition and the two-of-three containers condition. Consistent with the results of our previous studies, participants in the two-of-two containers condition were more likely to transcribe for the full ten minutes $(69.1 \%)$ than were 
participants in the combined containers condition $(46.5 \%$; $=-0.94, \mathrm{SE}=.45$, Wald $=4.33$, $\operatorname{Exp}(\mathrm{B})=0.39, p=.04)$ or the two-of three containers condition $(26.1 \%), \mathrm{B}=-1.84, \mathrm{SD}=$ .47, Wald $=15.17, \operatorname{Exp}(B)=0.16, p=.01$. Participants in the combined containers condition were significantly more likely to work for the full ten minutes than were those in the two-ofthree containers condition, $\mathrm{B}=-0.90, \mathrm{SD}=.45, \mathrm{Wald}=3.94, \operatorname{Exp}(\mathrm{B})=0.41, p=.05$.

Anticipated regret. We conducted a one-way ANOVA to examine whether condition affected anticipated regret and found a marginally significant effect, $F(2,127)=2.40, p=.09$. Participants in the two-of-two containers condition indicated that they would experience greater regret for their decision if they only worked for five minutes and therefore did not receive a second reward $(M=4.22, S D=1.49)$ than did participants in the other two conditions, $(M=3.63, S D=1.61), t(83.6)=2.02, p=.05, d=.38$. Participants in the two-oftwo containers condition anticipated significantly greater regret than did participants in the two-of-three containers condition $(M=3.48, S D=1.57) ; t(84.7)=2.25, p=.03, d=.48$. The contrast between the two-of-two containers condition and the combined containers condition did not reach significance $(M=3.79, S D=1.66), t(81.7)=1.25, p=.22, d=.27$.

As shown in Figure 2, anticipated regret mediated the relationship between being in the two-of-two containers condition vs. being in the other two conditions and increased likelihood of transcribing for the full ten minutes ( $95 \%$ bootstrapping confidence intervals of indirect effects, $L L=.002, U L=.580)$.

Experimenter concern. Our categorization manipulation did not affect how much participants thought the experimenter cared about how much they transcribed (all $p s>.55$ ).

\section{Discussion}

The results of Experiment 6 provide further evidence for the link between dividing rewards into categories and increased motivation, even when the categories are meaningless. Furthermore, this experiments shows that the anticipated regret associated with missing out 
on a reward explains the relationship between categorizing rewards and heightened effort in pursuit of those rewards. As in Experiment 3, the results are not consistent with a distinct reference point explanation for the link between categorizing rewards and enhanced effort.

\section{General Discussion}

Across six experiments, assigning incentives to distinct categories increased participants' motivation to exert effort on a variety of tasks. Even if we arbitrarily constructed the distinctions among categories of potential rewards, participants spent significantly more time working on tasks when they were told that the benefits of doing so would stem from multiple categories than they did when the benefits were not said to stem from multiple categories. These effects proved robust under various conditions. First, they occurred even though participants who worked to attain non-categorized incentives could obtain some or even every possible combination of rewards that participants in the categorization condition could obtain. Second, the effects also occurred despite the fact that participants visually inspected the items prior to working on the given task and, in Experiment 2, estimated their monetary value. Lastly, they also occurred when exactly the same rewards were present in each category and when there was no explicit mention of categories.

The results of our studies also provide evidence for the psychological mechanism explaining this categorization-of-rewards effect. We found that the regret participants anticipated that they would experience as a result of potentially missing out on a second reward mediated the effects of categorization on increased effort. Our results also indicated that categorization of rewards did not lead to enhanced effort if such effort did not allow participants to ameliorate their anticipated regret about missing out by obtaining a reward from each available category. Our findings are therefore consistent with a fear-of-missing-out mechanism and do not provide support for the alternative possibility that categorization could 
lead to enhanced effort by leading people to establish distinct reference points to be used in the evaluation of the utility derived from the reward.

\section{Theoretical Contributions}

The present research contributes to the extant literature on motivation, to psychology research on the effects of creating categories, and to behavioral decision research in several ways. First, our studies are the first empirical investigation that demonstrates a link between categories of rewards, anticipated regret, and motivation. In doing so, our work adds to the collective understanding of how dividing rewards into categories can affect when and where individuals are likely to invest their efforts, even when those categories are completely arbitrary. Our findings may thus offer people a novel way to motivate themselves and avoid procrastinating. By giving themselves rewards that they can classify into distinct categories, people may find the increased motivation they need to perform the tasks necessary to accomplish their goals.

Second, our results are unique in showing that mental segregation of rewards can increase the regret people anticipate they will experience by missing out on a potential reward. As we have shown through various analyses, people became more motivated to apply effort in pursuit of a second reward when their effort could ameliorate the regret they anticipate that they will experience from missing out on a reward.

Our research also suggests that fear of missing out may influence people's decisions to invest resources other than effort. Heath (1995) has found that people become hesitant to escalate their commitment beyond pre-set budgets unless they can escalate their commitment by drawing resources from a category of resources that was not initially budgeted. For example, after spending all of the money allotted to a project, those involved would be more likely to invest additional time than additional money. In contrast, if they were almost out of time, they would be more likely to invest additional money than additional time. In short, 
when the costs come from multiple categories, people can more easily ignore the fact that they are escalating their commitment beyond the level they initially intended. In contrast, our work focuses on the benefits of goal attainment. It suggests that a goal that comes with multiple categories of benefits may lead people to be more likely to escalate their commitment than would a goal that produces benefits that are not categorized. Future research could productively examine whether categorization of benefits systematically affects escalation of commitment.

Importantly, our findings also contribute to existing work on individual motivation. Prior work in psychology has examined the factors that increase motivation and the processes that underpin motivation from various perspectives. One theoretical approach has been particularly influential: the organismic approach (Deci \& Ryan, 1985). This approach focuses on the contexts and dispositional orientations that affect motivation. For instance, according to one of the well-known models in this tradition, namely self-determination theory (Deci \& Ryan, 1985), individuals are guided by two main types of motivations, controlled and autonomous, which are influenced by various environmental factors. Our work extends this body of research by focusing on a previously overlooked factor: creating categories of rewards. We demonstrated that simply dividing rewards into multiple categories increases the effort people exert on tasks. Across six experiments, we found that even when the categories of rewards are completely arbitrary, they enhance individual motivation to work harder on the given task.

The present research also contributes to extant behavioral decision research that demonstrates that the mere presence of seemingly irrelevant factors influences individual decisions and behaviors. For instance, prior work has identified a mere accessibility effect, by which making positive information easy to retrieve triggers more positive evaluations (Menon \& Raghubir, 2003). Closer to the current investigation, Mogilner and colleagues 
(2008) have identified a mere categorization effect in consumer behavior, whereby the presence of category labels in an option display increased consumer satisfaction with a product. Extending this body of work, our research focused on the effects of categorizing rewards and investigated how such categorization, even when completely arbitrary and meaningless, can influence individual motivation to exert effort on tasks by heightening people's fear of missing out on potential rewards.

\section{Limitations and Directions for Future Research}

The contributions of our research must be qualified in light of several limitations. First, we did not investigate the effects of creating meaningful or meaningless categories on motivation when people face tasks that are clearly intrinsically interesting or when they have the opportunity to learn. The task that comes closest to these types of task is the editing task used in Experiment 5. Although we believe our results would generalize to those contexts (as suggested by the results of Experiment 5), future studies would benefit from examining how creating categories of rewards can stimulate motivation for tasks that are intrinsically motivating or that provide an opportunity for learning.

Second, we did not examine the role of dispositional factors as potential moderators for the effect of creating categories of rewards on individual motivation. For instance, need for achievement (McClelland, Atkinson, Clark, \& Lowell, 1958) may moderate the categorization effect observed in our studies, as people who are high in need for achievement may feel particularly compelled to "tick off all the boxes" and obtain all categories of rewards. Future research could also investigate the moderating role of situational rather than dispositional factors. For instance, one could examine whether individuals are more sensible to these categorization effects when they are working under time pressure or when they are competing with others who can obtain rewards from the same set of categories. 
Third, our research has only focused on the effects of creating categories of incentives that serve as rewards for individual performance. Categorizing rewards may not reliably increase motivation in all types of relationships. Increasing the magnitude of compensation reliably increases effort in monetary markets but does not reliably produce the same increase in effort within relationships that are not based on monetary exchange (i.e., social markets) (Heyman \& Ariely, 2004). Future work could therefore examine if categorizing rewards increases motivation in both social and monetary markets to the same degrees. Future studies could also explore whether categorizing rewards can, in some instances, transform people's conceptualization of an exchange relationship from a social market to a monetary market. If this is the case, then categorizing rewards could actually decrease effort, as the introduction of a monetary form of payment can sometimes reduce motivation relative to conditions in which no payment of any kind is offered (Heyman \& Ariely, 2004).

In this research, we focused on how exogenously creating categories of rewards for performance influences motivation and effort. Future research could examine how exogenously creating categories of goals affects motivation and effort. Recent research has highlighted the important role of self-regulation in goal pursuit and motivation (e.g., Fishbach \& Dhar, 2005; Fishbach, Dhar, \& Zhang, 2006) and has demonstrated that the course of selfregulation over time depends on whether people are asked about commitment or about progress. Future work could investigate whether dividing goals into categories, even when arbitrary, can help individuals track their progress, and more easily achieve their goals.

Finally, our research has focused primarily on the beneficial effects of the presence of categories of rewards on individual motivation. Yet, categories of rewards may also lead to costly behavior or suboptimal decisions. For instance, people may continue engaging in the same course of actions because they are motivated to obtain a reward from a different category even when the course of action is not beneficial (as in the case of escalation of 
commitment or sunk costs). Our work has started investigating the powerful effects that meaningless categories can have on motivation. Future research extending our work to domains where the categorization effect may be costly could further our understanding of the effects demonstrated here.

\section{Conclusion}

In this paper, we proposed that separating rewards into categories increases motivation, even when those categories are completely arbitrary and meaningless. Across six laboratory experiments employing different categories of rewards, as well as different tasks and measures of effort, we found robust support for this prediction. Our results are important in light of the fact that fostering human motivation has been a topic of interest across disciplines over the last fifty years, and one of clear practical relevance. Our research suggests that taking existing possible rewards and splitting them into categories is a simple way to bolster individual motivation. 


\section{References}

Atkinson, J. W. (1964). Introduction to motivation. Princeton, NJ: Van Nostrand.

Battalio, R. C., Kagel, J. H., \& Jiranyakul, K. (1991). Testing between alternative models of choice under uncertainty: Some initial results. Journal of Risk and Uncertainty, 3, 2550.

Bell, D. E. (1982). Regret in decision making under uncertainty. Operations Research, 30, 961-981.

Bell, D. E. (1983). Risk premiums for decision regret. Management Science, 29, 1156-1166.

Clark, H. H. (1985). Language use and language users. In Gardner Lindzey and Elliot Aronson (Eds.). Handbook of Social Psychology, New York: Harper \& Row (pp. 179-231).

Deci, E. L., \& Ryan, R. M. (1985). Intrinsic motivation and self-determination in human behavior. New York: Plenum.

Fishbach, A., \& Dhar, R. (2005). Goals as excuses or guides: The liberating effect of perceived goal progress on choice. Journal of Consumer Research, 32(3), 370-377.

Fishbach, A., Dhar, R., \& Zhang, Y. (2006). Subgoals as Substitutes or Complements: The Role of Goal Accessibility. Journal of Personality and Social Psychology, 91, 232242.

Grant, A. M. (2007). Relational job design and the motivation to make a prosocial difference. Academy of Management Review, 32(2), 393-417.

Grant, A. M. (2008). The significance of task significance: Job performance effects, relational mechanisms, and boundary conditions. Journal of Applied Psychology, 93(1), 108-124.

Grant, A. M., Campbell, E. M., Chen, G., Cottone, K., Lapedis, D., \& Lee, K. (2007). Impact and the art of motivation maintenance: The effects of contact with beneficiaries on 
persistence behavior. Organizational Behavior and Human Decision Processes, 103, $53-67$.

Grice, H. P. (1975). Logic and conversation. In Peter Cole and Jerry L. Morgan (Eds.),Syntax and Semantics III: Speech Acts, New York: Academic Press (pp. 41-58).

Hackman, J. R. \& Oldham, G. R. (1976). Motivation through the design of work: test of a theory. Organizational Behavior and Human Decision Processes, 16(2), 250-279.

Harless, D. W. (1992). Actions versus prospects: The effect of problem representation on regret. American Economic Review, 82, $634-649$.

Heath, C. (1995). Escalation and de-escalation of commitment in response to sunk-costs: the role of budgeting in mental accounting. Organizational Behavior and Human Decision Processes, 62, 38-54.

Heit, E., \& Rubinstein, J. (1994). Similarity and property effects in inductive reasoning. Journal of Experimental Psychology: Learning, Memory, and Cognition, 20, 411-422.

Heyman, J., \& Ariely, D. (2004). Effort for payment: A tale of two markets. Psychological Science, 15(11), 787-793.

Kahneman, D., \& Tversky, A. (1979). Prospect theory: An analysis of decision under risk. Econometrica, 47, 263-291.

Kahneman, D., \& Tversky, A. (1981). The framing of decisions and the psychology of choice. Science, 211, 453-458.

Kandell, J. (1998). Internet addiction on campus: The vulnerability of college students. CyberPsychology \& Behavior, 1(1), 11-17.

Killeen, P. R. (1979). Arousal: Its genesis, modulation, and extinction. In M. D. Zeiler \& P. Harzem (Eds.), Advances in analysis of behaviour: Vol. 1. Reinforcement and the organization of behaviour (pp. 31-78). Chichester, England: Wiley. 
Killeen, P. R. (1982). Incentive theory: II. Models for choice. Journal of the Experimental Analysis of Behavior, 38, 217-232.

Kindleberger, C. P. (1978/1989). Manias, Panics, and Crashes: A History of Financial Crises. Rev. ed. NewYork: Basic Books.

Koestner, R., \& Losier, G. F. (2002). Distinguishing three ways of being internally motivated: A closer look at introjection, identification, and intrinsic motivation. In E. L. Deci \& R. M. Ryan (Eds.), Handbook of self-determination research (pp. 101121). Rochester, NY: University of Rochester Press.

Laffont, J. J., \& Martimort, D. (2002). The Theory of Incentives: the Principal-Agent Model. Princeton University Press: Princeton.

Larrick, R. P. \& Boles, T. L. (1995). Avoiding regret in decision with feedback: A negotiation example. Organizational Behavior and Human Decision Processes, 63, 87-97.

Lassaline, M. E. (1996). Structural alignment in induction and similarity, Journal of Experimental Psychology: Learning, Memory, and Cognition, 22, 754-770.

Lewin, K. (1935). A dynamic theory of personality: Selected papers. New York: McGraw Hill (D. E. Adams \& K. E. Zener, Trans).

Locke, E. A. (1968). Toward a theory of task motivation and incentives. Organizational Behavior and Human Performance, 3(2), 157-189.

Locke, E. A., \& Latham, G. P. (2002). Building a practically useful theory of goal setting and task motivation: A 35 year odyssey. American Psychologist, 57, 705-717.

Loomes, G. (1988). Further evidence of the impact of regret and disappointment on choice under uncertainty. Economica, 55, 47-62.

Loomes, G., Starmer, C., \& Sugden, R. (1992). Are preferences monotonic? Testing some predictions of regret theory. Economica, 59, 17-33. 
Loomes, G., \& Sugden, R. (1982). Regret theory: An alternative theory of rational choice under uncertainty. Economic Journal, 92, 805-824.

MacKinnon, D. P., \& Dwyer, J. H. (1993). Estimating mediated effects in prevention studies. Evaluation Review, 17(2), 144-158.

MacKinnon, D. P., Fairchild, A. J., \& Fritz, M. S. (2007). Mediation analysis. Annual Review of Psychology, 58, 593-614.

Mazar, N., Amir, O., \& Ariely, D. (2008). The dishonesty of honest people: A theory of selfconcept maintenance, Journal of Marketing Research, 45(6), 633-644.

McClelland, D. C., Atkinson, J. W., Clark, R. A., \& Lowell, E. L. (1958). A scoring manual for the achievement motive; R. W. Heyns, J. Veroff, \& J. W. Atkinson, A scoring manual for the affiliation motive; J. Veroff, A scoring manual for the power motive. Respectively, Chapters 12, 13 and 14 in J. W. Atkinson (Ed.), Motives in Fantasy, Action and Society. New York: Van Nostrand.

McDowell, J. J, \& Kessel, R. A. (1979). A multivariate rate equation for variable-interval performance. Journal of the Experimental Analysis of Behavior, 31, 267-283.

Menon, G., \& Raghubir, P. (2003). Ease-of-retrieval as an automatic input in judgments: A mere accessibility framework? Journal of Consumer Research, 30, 230-243.

Mogilner, C., Rudnick, T., \& Iyengar, S. S. (2008). The mere categorization effect: How the presence of categories increases choosers' perceptions of assortment variety and outcome satisfaction. Journal of Consumer Research, 35, 202- 215.

Preacher, K. J., \& Hayes, A. F. (2004). SPSS and SAS procedures for estimating indirect effects in simple mediation models. Behavior Research Methods, Instruments, and Computers, 36, 717-731.

Ritov, I. (1996). Probability of regret: Anticipation of uncertainty resolution in choice. Organizational Behavior and Human Decision Processes, 66, 228-236. 
Rosch, E. (1973). Natural categories. Cognitive Psychology, 4(3), 328-350.

Rosch, E. (1975). Cognitive reference points. Cognitive Psychology, 7, 532-547.

Rosch, E. (1978). Principles of categorization. In Rosch, E. and Lloyd, B.B. (eds), Cognition and categorization (pp. 27-48) Lawrence Erlbaum Associates, Publishers, (Hillsdale).

Rosch, E. (2002). Principles of categorization. Foundations of Cognitive Psychology: Core Readings, ed. Daniel Levitin, Cambridge, MA: MIT Press, 251-270.

Rosch E., \& Lloyd, B. B. (1978). Cognition and categorization. Hillsdale, NJ: Lawrence Erlbaum Associates, Inc.

Rosch, E., Mervis, C. B., Gray, W. D., Johnson, D. M., \& Boyes-Braem, P. (1976). Basic objects in natural categories, Cognitive Psychology, 8, 382-439.

Rutherford, S. (2001). 'Are you going home already?': The long hours culture, women managers and patriarchal closure. Time and Society, 10, 259-276.

Ryan, R. M., \& Deci, E. L. (2000). Self-determination theory and the facilitation of intrinsic motivation, social development, and well-being. American Psychologist, 55, 68-78.

Sansone, C., \& Harackiewicz, J. M. (2000). Intrinsic and extrinsic motivation: the search for optimal motivation and performance. Amsterdam: Elsevier.

Schweitzer, M., Ordonez, L. \& Douma, B. (2004). Goal setting as a motivator of unethical behavior. Academy of Management Journal, 47(3), 422-432.

Simonson, I. (1992). The influence of anticipating regret and responsibility on purchase decisions. Journal of Consumer Research, 19, 105-118.

Sloutsky, V. (2003). The role of similarity in the development of categorization. Trends in Cognitive Sciences, 7, 246-251.

Thaler, R. H. (1985). Mental accounting and consumer choice. Marketing Science, 4, 199214. 
Thaler, R. H. (1990). Saving, fungibility and mental accounts. Journal of Economic Perspectives, 4, 193-205.

Thaler, R. H. (1999). Mental accounting matters. Journal of Behavioral Decision Making, $12(3), 183-206$.

Thaler, R. H., \& Johnson, E. J. (1990). Gambling with the house money and trying to break even: The effects of prior outcomes on risky choice. Management Science, 36(6), 643-660.

Tracey, J. (2003). The emotional response to the injury and rehabilitation process. Journal of Applied Sport Psychology, 15(4), 279-293

Vroom, V. H. (1964). Work and motivation. New York: Wiley.

Zeelenberg, M. (1999). Anticipated regret, expected feedback, and behavioral decision making. Journal of Behavioral Decision Making, 12(2), 93-106.

Zeelenberg, M. \& Beattie, J. (1997). Consequences of regret aversion 2: Additional evidence for effects of feedback on decision making. Organizational Behavior and Human Decision Processes, 72, 63-78.

Zeelenberg, M., Beattie, J., van der Pligt, J. \& de Vries, N. K. (1996). Consequences of regret aversion: Effects of expected feedback on risky decision making. Organizational Behavior and Human Decision Processes, 65, 148-5.

Zeelenberg, M., van Dijk, W. W., \& Manstead, A. S. R. (1998). Reconsidering the relation between regret and responsibility, Organizational Behavior and Human Decision Processes, 74, 254-72. 
Tables

Table 1

Participants' effort and motivation by condition, Experiment 2

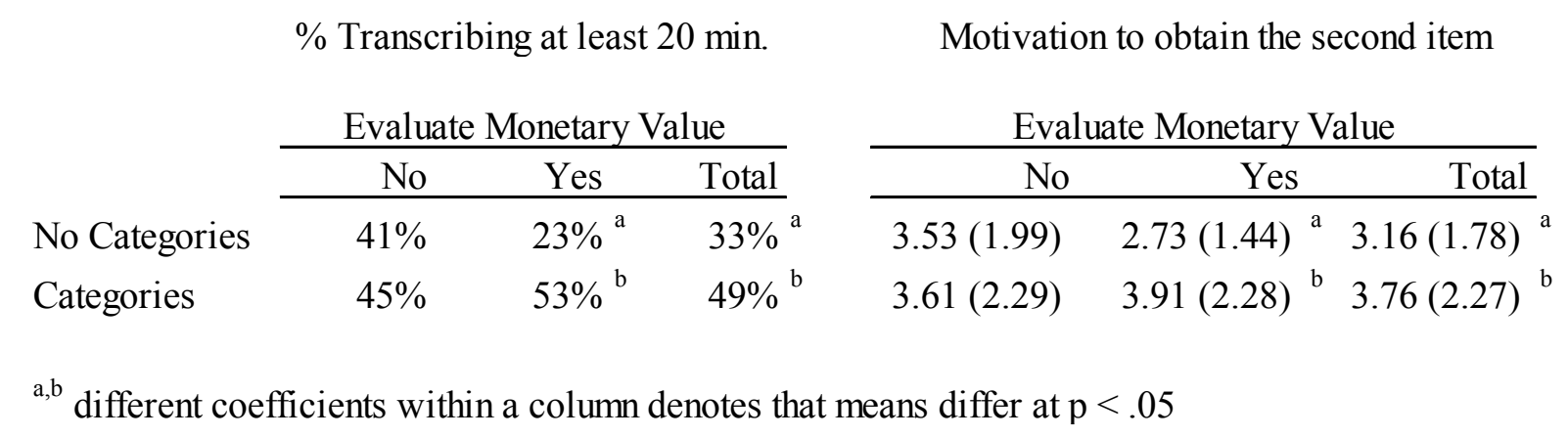


Figures

Figure 1: Value function

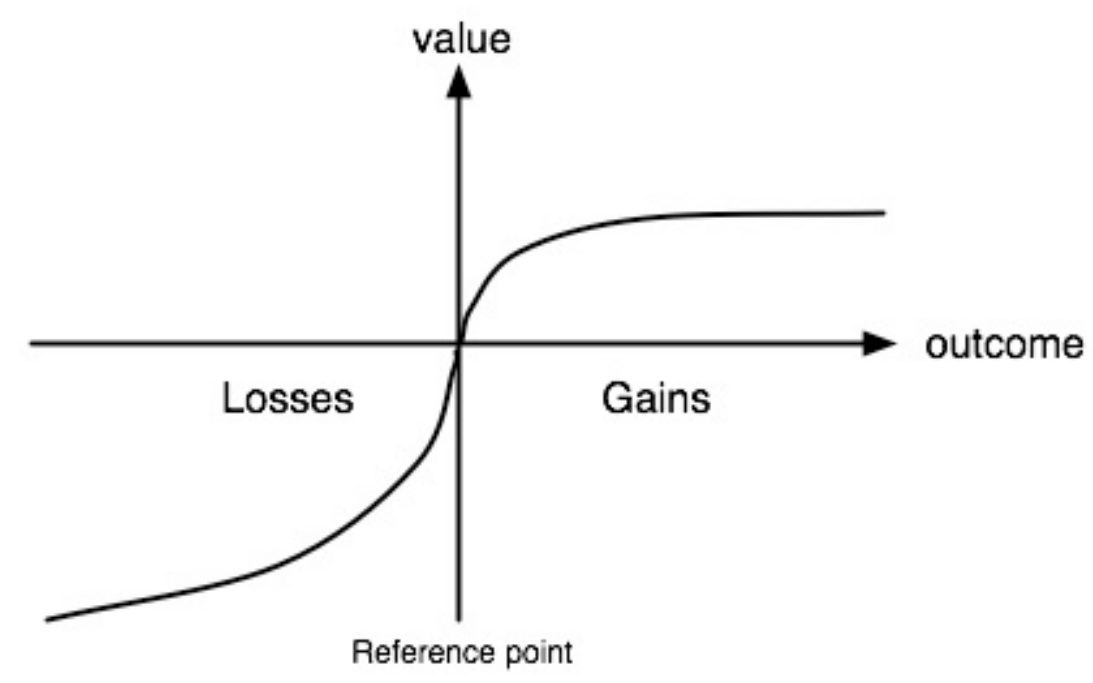

Figure 2: Anticipated regret mediates the relationship between categorized rewards and likelihood of transcribing text for the full ten minutes (Experiment 6)

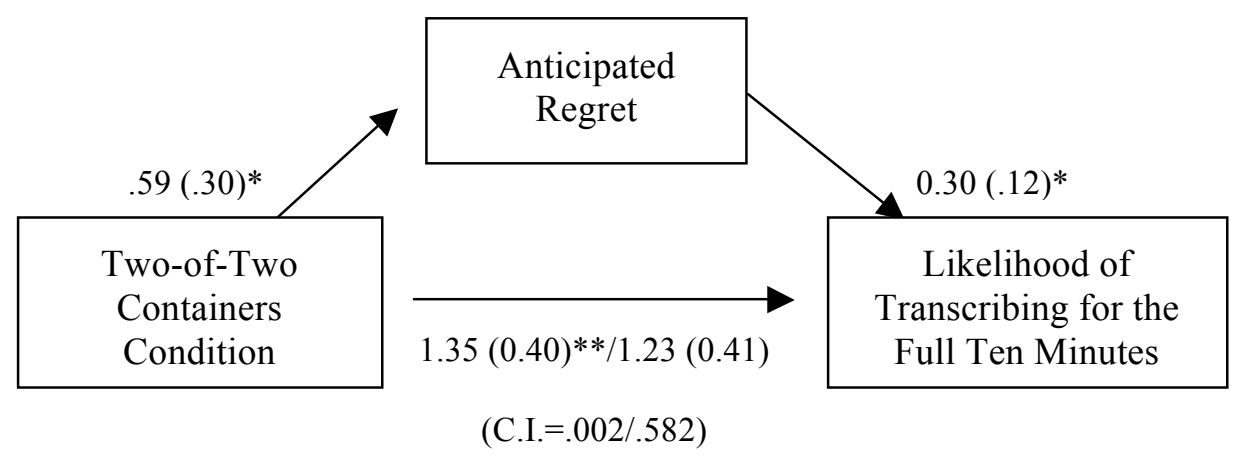

Values are unstandardized regression coefficients (values in parentheses are standard errors). 